\title{
Lexis
}

Journal in English Lexicology

Book reviews | 2021

\section{Sandrine SORLIN (dir.), Inventive Linguistics}

Presses universitaires de la Méditerranée, 2010, 212 pages

\section{Stéphanie Béligon}

\section{(2) OpenEdition}

\section{Journals}

Édition électronique

URL : https://journals.openedition.org/lexis/5134

DOI : $10.4000 /$ lexis. 5134

ISSN : 1951-6215

\section{Éditeur}

Université Jean Moulin - Lyon 3

Référence électronique

Stéphanie Béligon, «Sandrine soruin (dir.), Inventive Linguistics», Lexis [En ligne], Recensions, mis en ligne le 21 mai 2021, consulté le 23 mai 2021. URL : http://journals.openedition.org/lexis/5134 ; DOI : https://doi.org/10.4000/lexis.5134

Ce document a été généré automatiquement le 23 mai 2021.

\section{(c) (i) (9)}

Lexis is licensed under a Creative Commons Attribution-NonCommercial-NoDerivatives 4.0 International License. 


\title{
Sandrine SORLIN (dir.), Inventive Linguistics
}

Presses universitaires de la Méditerranée, 2010, 212 pages

\author{
Stéphanie Béligon
}

\section{RÉFÉRENCE}

\section{Sandrine Sorlin}

Inventive Linguistics. Presses universitaires de la Méditerranée, Collection « Sciences du langage », Montpellier, 2010. ISBN : 978-2-84269-908-6, Prix : 21€, 212 pages

1 Cet ouvrage de 212 pages fait suite au colloque interdisciplinaire tenu à l'Université Paul Valéry-Montpellier 3 en mars 2009 sur la thématique "Inventive Linguistics». Sandrine Sorlin rappelle dans l'introduction que l'une des idées fondatrices du colloque était d'interroger la validité de la notion d'« invention » en linguistique dans la mesure où le langage précède les locuteurs: la langue n'est-elle pas plutôt un ensemble de règles et de contraintes qui s'impose toujours déjà à eux ? L'auteure note toutefois la capacité des locuteurs à enfreindre ces règles. Par ailleurs, l'invention linguistique est collective, puisqu'une création ne peut survivre que si elle est acceptée et adoptée par une communauté linguistique. Ce sont notamment ces phénomènes, habituellement délaissés par la linguistique, que prendrait pour objet une linguistique inventive, qui serait donc plus inclusive que la linguistique «traditionnelle » et prendrait en compte les forces centrifuges qui conduisent aux changements linguistiques.

2 L'ouvrage s'organise en quatre chapitres: le premier, "Alienation, Renunciation, Imagination » se constitue des contributions de Jean-Jacques Lecercle, Sandhya Patel, Andy Arleo et Anett Jessop, qui examinent comment remédier à l'aliénation linguistique à laquelle tout locuteur est soumis : celle de ne pas pouvoir exprimer ce qu'il veut vraiment dire. Le deuxième chapitre, "Re-Reading, Re-Defining, ReInventing ", qui traite de la linguistique comme discipline et des présupposés qui la sous-tendent, regroupe les contributions de Gérard Mélis, Sandrine Sorlin et John 
E. Joseph. Dans le troisième chapitre, "Reform, Usage and Identity », Laure Gardelle, Gilles Siouffi et Gilles Chamerois se demandent dans quelle mesure les locuteurs peuvent transformer la langue qu'ils utilisent. Dans le quatrième et dernier chapitre, «Creativity and Inventiveness " Judith Munat s'intéresse à la créativité lexicale, Jim Walker étudie la distinction entre créativité, liée à l'application de règles productives, et inventivité, Denis Jamet examine les métaphores relatives à la mort comme moyen d'inventer une réalité " désodorisée », et Jocelyn Dupont analyse l'emploi inventif du style chez Edgar Allan Poe pour dépeindre la folie.

3 Le premier chapitre s'ouvre sur la contribution de Jean-Jacques Lecercle, intitulée «Linguistic alienation, linguistic imagination and literary solipsism» (p. 19-33); cet article soulève la question de ce qu'est l'aliénation linguistique et de comment la surmonter: l'imagination linguistique est-elle un moyen d'y parvenir? L'aliénation linguistique est constitutive de la langue puisque celle-ci est un système qui s'impose au sujet parlant. Pourtant, cette contrainte est aussi féconde, puisque la langue est le seul moyen de sortir de l'état d'infans. Par ailleurs, toutes les règles, qu'elles soient phonétiques, lexicales ou syntaxiques, peuvent être remises en cause par la créativité des locuteurs. On peut donc voir dans ces contraintes, à la suite de J. Butler [1997], des voies d'émancipation (« empowerment »). Jean-Jacques Lercecle s'intéresse ensuite plus précisément à la façon dont la littérature exploite cette contradiction inhérente au langage et identifie trois stratégies: "weirding language", le solipsisme littéraire ( literary solipsism ») et l'imagination linguistique.

4 La désaliénation par la littérature peut reposer sur la construction d'un canon de textes, tels que ceux de Proust, Kafka, Artaud, Beckett ou Ghérasim Luca, mais elle relève alors de la culture occidentale et n'a qu'une portée politique limitée. "Weirding English » apparaît dès lors comme une stratégie plus radicale : le concept de "weird English », exposé par E. Ch'ien [2004], se fonde sur l'observation d'écrivains polyglottes comme Nabokov, Maxine Hong Kingston, Arundhati Roy ou Junot Diaz. Le « weird English » ôte, selon Evelyn Ch'ien, sa position dominante à l'anglais. Il inclut également l'anglais du West Country, par exemple, ou l'anglais écossais employé dans Trainspotting, où le "weirding " a aussi une dimension sociale. Or c'est là une des limites de cette forme de désaliénation puisque, dans la plupart des cas, l'écrivain ou l'écrivaine qui prétend écrire au nom d'un groupe marginalisé ne fait que renvoyer au groupe dominant l'image que celui-ci se fait du groupe dominé. De ce point de vue, le "weirding " échoue dans sa tentative de désaliénation.

5 Le solipsisme littéraire, tel que celui de Beckett, assume la nature intrinsèquement aliénante du langage. Jean-Jacques Lecercle s'appuie sur le passage suivant :

comment c'était je cite avant Pim avec Pim après Pim c'est trois parties je le dis comme je l'entends

voix d'abord dehors quaqua de toutes parts puis en moi quand ça cesse de haleter raconte-moi encore finis de le raconter invocation [S. Beckett : $1961: 9$ ]

et souligne que par l'emploi de «quaqua», le locuteur imite la langue («Quoi ? Quoi ?») mais ce «mot» s'apparente aussi au cri qui perturbe l'ordre du langage articulé, dénonce l'aliénation induite par le «bavardage » et les clichés, et s'en libère. L'imagination linguistique est donc un ressort de la désaliénation. Elle est aussi exploitée dans les langues imaginaires (cf. Riddley Walker, de Russell Hoban) ou par des poètes comme Alex Dickown, auteur de poésie bilingue qui bafoue les règles de la syntaxe : 


\begin{tabular}{|l|l|}
\hline You are worn a raincoat & C'est l'imperméable \\
\hline who I was giving you away & je porte dont tu m'as laissé \\
\hline to keep you out and escape & pour m'échapper la pluie \\
\hline you from the rain when it's & de tomber sur moi et \\
\hline onto you, or when you're & pour éviter les nuages \\
\hline spotting clouds after you & à l'horizon me menacer \\
\hline from nearby & the horizon [A. Dickow 2008:32-33] \\
\hline
\end{tabular}

Son emploi "fautif» de la langue fait ainsi émerger d'autres formes d'anglais et de français et conduit de l'aliénation linguistique à une poétique de l'imagination littéraire et linguistique.

Dans l'article suivant, «Inventive Linguistics: Albert Wendt's Pacific Sites of Creation » (p.35-45), Sandhya Patel s'intéresse à un roman d'Albert Wendt publié en 2003, The Mango's Kiss, qui mêle le samoan et l'anglais, comme l'illustre le passage suivant :

In the neighbouring fale and circling the malae in the shade were those who'd come with the guests - hundreds of them. The hungry air was thick with the smell of the umu-cooked pork, chicken, fish, palusami, faiai, taro, ota, faiai fe'e, povi masima, pisupo and other dishes. Throughout the previous night nearly al the aiga in Malelua had made umu for the feast. [Wendt $2003:$ 45].

Sandhya Patel observe que les mots samoans sont fréquents dans le roman et produisent un effet de défamiliarisation qui diffère la compréhension du texte, ce qui, d'une part, met en lumière des spécificités culturelles samoanes et, d'autre part, contribue à la création d'un objet littéraire esthétique. À la différence d'autres romans antérieurs d'Albert Wendt, dans The Mango's Kiss, les termes samoans s'insèrent dans le texte sans guillemets ni italiques et remettent en cause le rythme de l'anglais. Par ailleurs, un terme tel que aiga, par exemple, acquiert ses diverses connotations au fil du roman, alors que les différents contextes dans lesquels il apparaît dévoilent le fonctionnement de la structure sociale qu'il désigne. Les couches de significations se superposent, ce qui remet constamment à plus tard la pleine compréhension du texte.

7 Sandhya Patel se demande si une linguistique inventive se déploie ainsi dans The Mango's Kiss par l'opacité qu'entretiennent les deux langues l'une envers l'autre: le roman cultive-t-il l'opacité pour résister à une transparence comme universalisme qui menace la vision du monde samoane et refuse au Pacifique la possibilité d'être un lieu de création littéraire ? Ou, au contraire, le mélange des deux langues est-il une forme de plus grande transparence?

Dans l'article intitulé «What happens when an invented language is set to music? A linguistic study of dogorian» (p.47-59), Andy Arleo étudie Dogora, suite populaire dogorienne de Proszeshny orientale, œuvre pour chœur et orchestre composée par Etienne Perruchon en 2000. Le dogorien, langue imaginaire, est "trompe l'oreille» «qui regrouperait toutes les influences vocales européennes au sens le plus large du terme ", selon le compositeur. L'enquête d'Andy Arleo se fonde sur un projet mené dans un collège de Saint-Nazaire, qui a donné lieu à trois représentations par des élèves de l'établissement, des chœurs adultes et des participants venus d'établissements partenaire basés en Ukraine, en Italie, en Allemagne et en Grande Bretagne.

Dans cette contribution, Andy Arleo décrit le dogorien, analyse la façon dont les choristes le perçoivent et s'intéresse à l'interaction entre langue et musique. Il soulève 
aussi des questions plus générales, telles que les suivantes: dans quelle mesure les langues existantes façonnent-elles les langues imaginaires? Est-il possible de créer une langue imaginaire dépourvue de signification?

10 Andy Arleo décrit les phonèmes, la structure syllabique et la prosodie du dogorien. Il s'intéresse dans un second temps aux résultats du questionnaire distribué aux choristes. Les questions posées (rappelées en annexe de l'article) portent sur le pouvoir d'évocation du dogorien, ses ressemblances avec des langues existantes, la signification de l'œuvre ou encore l'harmonie entre la musique et le dogorien. Andy Arleo montre en quoi les réactions des adultes et des élèves diffèrent quant à l'idée de chanter dans une langue imaginaire, comment les participants identifient cette langue, quelles significations ils attribuent aux paroles et quelles émotions ils leur associent.

11 L'auteur de l'article conclut que le dogorien ne peut pas être considéré comme une langue imaginaire à part entière, puisque, contrairement à l'espéranto, par exemple, il n'a pas une grammaire productive, ni de signification bien établie. Malgré tout, la plupart des enquêtés ont attribué un sens au texte et semblent même s'accorder sur les thèmes généraux. Bien que le programme ait pu avoir une influence sur leurs réponses, tout comme le discours d'Etienne Perruchon et le contexte local, ceci tend à prouver qu'il est presque impossible de créer une langue imaginaire dépourvue de sens, dans la mesure où les séquences de phonèmes tendent à être associées à des expériences linguistiques antérieures.

12 Anett Jessop, dans « Modernisms, Pure English, and Poetry: Laura (Riding) Jackson's “Linguistic Ultimate" » (p. 61-73), examine le cheminement de la poétesse Laura Riding, qui renonce en 1941 à la poésie, alors qu'elle écrivait encore en 1938 qu'un poème « is an uncovering of truth of so fundamental and general a kind that no other word besides poetry is adequate except truth ». Ainsi, alors qu'elle avait d'abord considéré que la langue poétique permettait d'accéder à la vérité, elle remet par la suite ce postulat en question.

13 À la fin des années 1930, face aux tensions politiques montantes en Europe, Laura Riding lance des campagnes de lettres pour mettre le langage au service de fins politiques. La nécessité d'une communication directe et sans équivoque lui fait prendre conscience que la poésie laisse une place trop grande aux erreurs d'interprétation : elle considère que les artifices poétiques nuisent à l'exactitude et se tourne vers la linguistique. Déjà, dans le poème «Come, Words, Away », publié en 1933, Riding semble évoquer l'échec de la poésie :

Come, words, away from mouths

Away from tongues in mouths, And reckless hearts in tongues And mouths in cautious heads Come, words, away to where The meaning is not thickened With the voice's fretting substance, Nor look of words is curious As letters in books staring out All that man ever thought strange And laid to sleep on white Like the archaic manuscript of dreams at morning blacked on wonder.

[Riding 1980 (1938) : 1-15] 
Anett Jessop observe que le poème invite les mots à quitter le monde de l'oralité, celui des bouches, des langues, de l'émotivité, pour un royaume où leur sens est révélé sans équivoque ni ambiguïté.

14 Au cours des années 1930, Laura Riding se lance dans la rédaction de thésaurus et de dictionnaires et incite Robert Graves, Jacob Bronowski, Eirlys Roberts ou encore Schuyler B. Jackson à se joindre à ses recherches concernant l'origine, le sens et les connotations des mots. Dans une préface à Rational Meaning, elle explique que l'un des objectifs de l'ouvrage est d'explorer «the nature of meaning itself» [L. R. Jackson \& S. B. Jackson 1997 : 17].

15 Pour Laura Riding, l'existence du langage découle de la nature humaine et sa fonction est d'ordonner la pensée pour exprimer la vérité (« ordering thought into the express forms we call truth» [L. R. Jackson \& S. B. Jackson $1997: 39])$. Les mots sont des entités intellectuelles ( intellectual entities »), leur sens ("meaning ») leur est inhérent et ne réside donc pas dans les locuteurs eux-mêmes, or c'est, selon elle, une idée dont les locuteurs n'ont plus conscience: "the realities of words as intellectual entities has become so pale in people's consciousness that, instead of knowing the meanings of their words, they know, rather, their habitual association-responses to the stimuli that the words in their physical actuality constitute" [L. R. Jackson \& S. B.Jackson 1997 : 49]. De plus, le désordre du monde s'explique selon elle parce que nous ne pensons plus de façon ordonnée et considérons que la vie humaine n'a pas de sens. Face à ce nihilisme, Laura Riding voit dans Rational Meaning un ouvrage porteur d'espoir ("a book of hope » [L. R. Jackson \& S. B. Jackson 1997 : 34]) : à ses yeux, l'effondrement de la responsabilité intellectuelle a mené à l'hégémonie de la méthode scientifique et à l'atomisation de la connaissance. Elle propose donc avec Rational Meaning une nouvelle approche du langage, "a charter of human rights to the dignity of a speech of unlimited truth, and a declaration of linguistic independence from ideas of language that enslave the mind to other laws than those of it natural relation with its words » [L. R. Jackson \& S. B. Jackson $1997: 8$ ].

C'est la contribution de Gérard Mélis, «Parole - discourse - enunciative processes: return, renewal and invention" (p. 77-84), qui ouvre le deuxième chapitre. L'auteur analyse les liens qui unissent les textes de Saussure, Benveniste et Culioli, bien que ces trois auteurs paraissent a priori s'inscrire dans des perspectives différentes.

17 Selon Saussure, seule la langue, en tant que système de signes, peut être étudiée, à la différence de la parole, or l'opposition entre langue et parole laisse un vide, une « lacune » [Foucault 1994 (1969)] car la langue s'incarne dans la parole. Pour échapper à cette contradiction, Benveniste s'intéresse au mode sémantique, exprimé dans le discours, par opposition au mode sémiotique, qui concerne, quant à lui, le signe linguistique et ses propriétés formelles. À nouveau, Benveniste crée une «lacune » car il évacue l'objet commun au mode sémantique et au mode sémiotique qu'est le langage. Culioli, quant à lui, définit le langage comme une activité inconsciente régulée par des relations intersubjectives et qui produit des textes, des énoncés et des signes qui sont autant de traces de cette activité. En tant qu'activité, le langage est un phénomène global et non l'un des deux membres d'une dichotomie. Culioli reformule donc la proposition de Benveniste de façon plus radicale: le mode sémiotique, système de signes, est lui aussi le résultat de l'activité linguistique.

Selon Gérard Mélis, le mouvement qui mène de Saussure à Culioli en passant par Benveniste met en évidence un processus d'invention : la notion d'activité linguistique 
est implicite chez Saussure puisque celui-ci s'intéresse à des systèmes de signes une fois qu'ils sont constitués. Culioli renverse donc le texte de Saussure et prend pour objet central ce qui était délaissé par son prédécesseur.

Culioli forge également la notion d'«énonciateur", ensemble de coordonnées abstraites qui régulent les relations intersubjectives telles qu'elles sont marquées dans le système linguistique: l'énonciateur se distingue donc du locuteur. Cette notion émerge à partir de ce que Culioli conçoit comme une lacune dans le texte de Benveniste: l'«Ego » de Benveniste désigne à la fois un vrai sujet humain et une construction linguistique, ce qui conduit à des apories. La notion d'énonciateur permet de résoudre ces contradictions.

Saussure estime que la «parole" est une condition sine qua non de l'existence de la « langue » mais il ne dit pas comment sont reliées parole et langue. Dans l'approche de Benveniste, le mode sémiotique est présenté comme clairement distinct du mode sémantique mais le signe linguistique est une entité qui relève des deux systèmes, or Gérard Mélis remarque que la distinction n'est pas toujours aussi nette. Définir l'activité linguistique comme l'objet de la linguistique permet, en revanche, de dépasser les dichotomies "parole» / "langue» et mode sémiotique / mode sémantique. Le signe, qui fait figure de base stable dans l'optique de Saussure, est représenté come un processus par Culioli.

21 Gérard Mélis conclut sur l'idée que les dichotomies présentées par Saussure et Benveniste présentent l'intérêt de mettre en évidence la complexité du langage mais évacuent celui-ci comme objet d'étude. Elles laissent des lacunes, qui ouvrent des brèches pour formuler ce qu'elles n'avaient pas abordé.

22 Sandrine Sorlin, dans "Inventedness" and inventiveness: for a postmodern linguistics» (p. 85-94), souligne tout d'abord que l'idée selon laquelle la langue se détériore est une idée ancienne, qui prédate largement les SMS ou la messagerie instantanée. Au cours des siècles, de nombreuses tentatives de mettre un terme à l'évolution de la langue et à sa dégradation (que Sandrine Sorlin nomme « inventedness ») ont vu le jour, telles que les tentatives de rationalisation du XVIIe siècle dont le but est d'éliminer erreurs et malentendus. Les grammaires prescriptives visent également à préserver la langue de ceux qui l'emploient. De même, le politiquement correct vise à éliminer la densité historique de la langue et son évolution : c'est un effacement du passé. L'« inventedness » repose sur l'idée qu'il existe un modèle linguistique correct, qui rend illégitimes les autres modèles linguistiques.

Sandrine Sorlin observe que l'idée d'un modèle linguistique correct a souvent eu pour effet de reléguer au rang d'excentricités les créations linguistiques dans la littérature, alors que celles-ci exploitent pleinement le potentiel de la langue comme processus, ainsi que l'illustrent les écrits de science-fiction d'Anthony Burgess, Russell Hoban, Will Self ou David Mitchell : paradoxalement, ces romanciers nous rapprochent de l'essence d'une langue en la défamiliarisant et l'inventivité («inventiveness ») consiste ainsi à mettre en évidence ce qui est déjà présent. C'est en ce sens que l'inventeur est un "bricoleur" de la langue qui emploie d'anciens mots dans un but nouveau et dépoussière des expressions toutes faites. Ce bricoleur linguistique nous pousse à voir sous un autre angle ce que nous avons toujours connu. On en arrive à un renversement complet de ce qui se présente au premier abord : les « excentriques littéraires » sont en réalité ceux qui sont le plus conformes à l'esprit de la langue, tandis qu'une version 
nationale standardisée est en réalité une construction politique, qui n'existe nulle part (cf. J -J. Lecercle [2004]).

Quant à la linguistique elle-même, elle pose problème en ce que l'énoncé est toujours le fruit d'influences collectives dont l'énonciateur n'est qu'un effet, et Sandrine Sorlin plaide pour une linguistique postmoderne qui inclue des aspects historiques, culturels, sociaux et politiques dont la langue ne peut être séparé. C'est la raison pour laquelle elle voit dans la linguistique littéraire une bonne candidate puisqu'elle fait du linguiste un interprète et non un propriétaire de la langue.

Dans "Hermeneiaphobia: why an "inventive" linguistics must first embrace interpretation» (p. 95-105), John E. Joseph souligne que la linguistique s'inscrit dans une tradition qui vise à identifier la bonne interprétation des textes et qui découle de l'idée qu'il est nécessaire de limiter la liberté des auditeurs et des lecteurs d'inventer des significations aux textes. Or, selon John E.Joseph, tenter de contrôler l'interprétation est voué à l'échec, puisque l'interprétation a précédé le langage et constitue même l'activité à laquelle s'est greffé le langage. L'interprétation, en tant que capacité à lire des données sensorielles présentes dans le monde comme autant de signes de quelque chose qui n'est pas immédiatement accessible à nos sens, est universelle au sens où elle concerne d'autres espèces vivantes que la nôtre. Les langues sont des traditions culturelles qui ont des fondements communs à de nombreuses autres espèces animales : les structures cérébrales et les dispositifs physiques impliqués dans la perception, la cognition et l'interprétation.

John E. Joseph se demande dans quelle mesure la linguistique contemporaine s'est éloignée de l'objectif premier de contrôler l'interprétation et note que les néoBloomfieldiens, qui ont dominé la scène de la linguistique aux États-Unis du milieu des années 1940 au début des années 1970, ont essayé d'éradiquer non seulement l'interprétation mais aussi le sens (cf. C. F. Hockett [1958]). Quant à Chomsky, il réduit l'interprétation à la parfaite transmission d'un contenu : à ses yeux, le co-locuteur se contente d'enregistrer passivement ce que le locuteur a créé.

John E. Joseph s'intéresse ensuite à un exemple d'inventivité linguistique, un texte Jacques de Voragine, qui propose une multitude d'étymologies du nom «Cecilia » dans La Légende dorée (1260) : il montre que Voragine fait ici preuve d'inventivité et que cette prolifération d'interprétations a été exposée à la résistance des lecteurs modernes qui la considèrent comme irrationnelle (cf. M. Carruthers [2000]).

28 L'auteur aborde ensuite l'œuvre de Whorf, à qui est souvent prêtée l'hypothèse que la structure de la langue d'un locuteur limite, voire détermine sa pensée, or John E. Joseph objecte que cela n'est pas le propos de ce linguiste, qui, loin de vouloir limiter l'interprétation, cherche au contraire à l'élargir : en s'appuyant sur l'expression du temps en hopi, Whorf montre qu'une langue peut masquer le réel et que d'autres expressions sont susceptibles d'ouvrir de nouvelles perspectives sur un réel donné.

29 John E. Joseph conclut en observant que, de nos jours encore, la linguistique s'évertue à mécaniser l'interprétation alors que, selon lui, une linguistique «inventive » doit se départir de son "herméneuphobie » et accueillir la dimension humaine de la langue, qui implique l'interprétation, toujours individuelle et parfois inventive.

30 Le troisième chapitre, "Reform, Usage and Identity ", inclut en premier lieu un article de Laure Gardelle, «Language reforms in English : gender in third-person pronouns » (p. 109-119). L'auteure souligne que l'histoire de l'anglais moderne est traversée par des 
tentatives régulières de changer l'emploi des pronoms de la troisième personne du singulier et elle prend pour exemples le débat sur le pronom utilisé pour désigner les bateaux.

31 Lloyd's List, revue consacrée aux transports maritimes, a tenté deux fois (en 1998 et en 2002) d'adopter le pronom it pour référer aux bateaux, au lieu de she, habituellement employé. Parmi les arguments invoqués pour justifier cette réforme, citons :

- celui de la modernité, puisque les autres organes de presse utilisent le pronom neutre pour renvoyer aux bateaux,

- la nécessité de l'emploi d'une langue non discriminatoire : l'idée sous-jacente est qu'il faut rapprocher le système des pronoms d'un fonctionnement purement sémantique, dans lequel la langue est une transposition directe du monde extralinguistique. Le genre grammatical est alors considéré comme le reflet d'une réalité sociale ou biologique.

Dans les deux cas, les réactions ont été vives : les opposants à cette réforme arguent de la tradition et de la dimension collective de la langue, qui invalident les volontés individuelles de modifier l'usage. De façon symétrique à celle des réformateurs, ils mettent également en avant l'idée que la langue est motivée et que l'emploi de she est donc certainement justifié, même si cela n'est plus manifeste pour les usagers contemporains de la langue.

Cette controverse soulève la question suivante: comment créer le besoin d'un changement linguistique ? Pour y répondre, Laure Gardelle prend l'exemple du pronom générique pour désigner les animés humains. Elle signale que la question d'un pronom épicène de troisième personne émerge dès le XVIIIe siècle, puis retrace l'histoire des pronoms épicènes de troisième personne: les pronoms inventés n'ont pas réussi à s'imposer dans la langue standard, pas plus que les pronoms it et one. L'emploi de she connaît davantage de succès mais se limite aux cercles universitaires et aux documents destinés aux parents de bébés ou de jeunes enfants. Ceci fait conclure à Laure Gardelle que la tendance qui s'impose est celle du recours à des pronoms existants plutôt qu'à des pronoms inventés.

L'auteure remarque que si les institutions sont nécessaires à la propagation des changements linguistiques, elles ne sont pas suffisantes car l'acceptation générale de la population est requise et la langue ne peut pas anticiper les changements culturels.

Gilles Siouffi, dans un article intitulé "How inventive could French $17^{\text {th }}$ century linguistics be?» (p. 121-133), note qu'au XVIIe siècle, la langue est considérée comme un objet philosophique qui doit être réformé pour pouvoir véhiculer des idées philosophiques. C'est également une période où de nombreux travaux de philologie ont été accomplis pour fixer les canons de la description des grammaires des langues modernes. Par ailleurs, le XVIIe siècle est aussi celui d'utopies linguistiques : Mersenne, Wilkins et Leibniz rêvent d'une langue philosophique universelle. C'est donc le siècle d'une «linguistique inventive", pourtant, il n'a abouti qu'à des changements linguistiques mineurs. Gilles Siouffi se propose donc, dans cette contribution, d'examiner les différents aspects de cette linguistique inventive et de montrer que la soumission à l'usage a finalement eu raison des innovations envisagées.

L'auteur s'intéresse d'abord à la création de langues imaginaires, telles que celles qu'inventent Cyrano de Bergerac dans Les États et Empires de la Lune (1659), Foigny dans Les Aventures de Jacques Sadeur dans la Découverte et le Voyage de la Terre Australe (1676) ou encore Vairasse d'Allais dans son Histoire des Sevarambes (1677 et 1678) : Gilles Siouffi 
observe que ces textes soulèvent les questions suivantes : les langues sont-elles liées à la nature ou à une institution? Sont-elles régies par la raison ou par l'usage ? L'usage est-il motivé ? Qui a autorité sur la langue?

Dans la deuxième partie de l'article, l'auteur s'intéresse d'ailleurs à la question de l'usage. La linguistique inventive de ce siècle se fonde sur le souhait d'instiguer un nouvel usage : Descartes, par exemple, s'interroge sur la possibilité de créer une langue universelle et considère qu'une langue idéale devrait reposer sur des idées philosophiques mais il estime qu'une telle langue ne sera jamais en usage. L'usage est également sacralisé par Vaugelas, qui le considère comme "maitre des langues vivantes » : « l'usage est celui auquel il se faut entièrement soumettre en notre langue, mais pourtant il n'en exclut pas la raison ni le raisonnement, quoiqu'ils n'aient nulle autorité » [C. F. de Vaugelas 1934 (1647)]. En réalité, Vaugelas concilie raison et usage par la croyance en la régularité de ce dernier.

Le troisième aspect inventif des travaux linguistiques du XVIIe siècle tient à ce que les descriptions linguistiques portent en réalité plutôt sur une version altérée de la langue, plus en accord avec une théorie ou un idéal que ne l'est l'usage attesté.

Gilles Siouffi conclut que les travaux linguistiques du XVIIe siècle avaient pour objectif de faire de la langue un vecteur d'émancipation mais que l'importance donnée à l'usage s'est révélée incompatible avec une ambition politique sur la langue. Par la création de langues artificielles purement abstraites (en mathématiques, logique ou informatique), le XXe siècle a réalisé un idéal du XVIIe (celui de des Vallées ou de Leibniz), mais l'emploi d'une langue désaliénante pour l'être humain ne s'est en revanche pas concrétisé.

Gilles Chamerois est l'auteur de "The "morphosis" of words in the "plexity" of the world: back-formation in Thomas Pynchon's Mason \& Dixon»(p.135-144). Dans le roman Mason \& Dixon (1997), des événements historiques et inventés des années 1760 sont narrés par un narrateur non fiable en 1786. Gilles Chamerois s'intéresse à la création lexicale dans ce roman: il commente par exemple l'emploi de l'adjectif rambunctious : "What we now style "The Stamp Act Crisis" was in full flower. [...] Thro' this rambunctious Countryside, a Coach-ful of assorted Travelers make their way Philadelphiaward, each upon his Mission" [T. Pynchon 1997:353], or l'emploi de rambunctious n'est attesté qu'à partir de 1859 ; le narrateur aurait en revanche pu avoir recours sans anachronisme à rumbustious (du latin robustus) employé en anglais britannique depuis 1778 : la liberté prise par l'auteur reflète l'indécision linguistique et territoriale qui caractérise l'époque à laquelle se déroule le récit.

Gilles Chamerois se penche aussi sur le mot jobate, qui semble à la fois être une dérivation régressive, à partir de jobation, et dériver par suffixation de jobe. Les troncations de ce type présentent la caractéristique d'inventer un nouveau passé tout en étant anachroniques, puisque le mot dont elles dérivent semblent en être le dérivé et donc succéder au néologisme qu'il précède. Ainsi, les lexèmes qu'étudie Gilles Chamerois constituent autant d'illustrations de questions de portée beaucoup plus vaste: rambunctious est la revendication de l'autonomie de l'anglais américain (en opposition à l'anglais britannique et au latin) et jobate suit sa Destinée manifeste en remplissant le vide laissé par jobe et jobate.

41 Gilles Chamerois souligne que ces mots constituent des pierres d'achoppement dans le texte, tout comme le mot 'Morphosis, qui, avec ses dérivés, présentent une dizaine d'occurrences dans le roman : à la fois opaques et transparents, ils dévoilent que les 
mots sont aussi des choses et que le langage est plus proche de la physique que de la métaphysique. Selon F. Ponge [2002 (1971): 431]: « les choses sont, déjà autant mots que choses et, réciproquement, les mots, déjà, sont autant choses que mots ».

Le quatrième et dernier chapitre, "Creativity and Inventiveness", s'ouvre sur la contribution de Judith Munat, «Creation or re-creation? Word formation processes and word creation strategies as components of lexical creativity» (p.147-157). L'auteure commence par définir la créativité linguistique qui, dans une perspective cognitive, réside selon elle dans la capacité de l'esprit humain à transformer des pensées en de nouvelles formes linguistiques. Elle distingue "forte créativité ", qui consiste à nommer de nouveaux concepts au moyen de lexèmes eux aussi entièrement nouveaux, et "faible créativité ", qui découle de notre capacité à combiner des éléments du lexique déjà existants pour former des mots, expressions ou phrases nouveaux ou originaux en appliquant des règles grammaticales. Judith Munat analyse, dans cet article, des exemples de "faible créativité » tirés de ses recherches antérieures. Elle a pour objectif de mettre en évidence les facteurs pragmatiques et sociaux qui influencent les choix lexicaux du locuteur dans un contexte donné.

Judith Munat rappelle d'abord les différents motifs qui président à la création lexicale : citons par exemple les composés déictiques («apple-juice seat» en vient ainsi à désigner une chaise en particulier, celle où se trouve le jus de pomme), les «dummy compounds », qui ont une dimension anaphorique (comme dans un énoncé tel que «We should talk about this vacation thing", où le composé renvoie à la connaissance partagée des locuteurs en présence) ou encore l'hypostasie, processus par lequel un nom est créé pour désigner un concept ou une entité non existant (" projecto-stylus ", "photonic dead zone », etc.). Ces nouvelles formations peuvent être utilisées dans un but humoristique, parodique ou encore ludique (dans la littérature jeunesse, par exemple).

44 Judith Munat rappelle que dans les textes de science-fiction qu'elle a étudiés antérieurement (cf. J. Munat [2007]), chaque auteur manifeste des préférences pour un procédé de formation lexicale plutôt qu'un autre, souvent en accord avec la thématique du roman. Elle note que les exemples de forte créativité sont plutôt rares dans la science-fiction, alors qu'ils prolifèrent dans le Big Friendly Giant de Roald Dahl (« buggles », « dillions », " glimp », « glummy », etc.), ce qui tient sans doute à l'intérêt phonologique qu'ils présentent pour un jeune public.

45 L'auteure s'intéresse ensuite à des "mots privés » (" private words ») inventés par des groupes restreints (cercles familiaux ou groupes d'amis). Elle note que ces mots consistent en des emprunts à des dialectes locaux ou à des langues étrangères, découlent d'erreurs de prononciation d'enfants, ou dérivent de noms propres. Selon elle, ce sont l'expérience commune et la connaissance partagée des locuteurs, ou encore la motivation phonologique qui sont à l'origine de ces inventions lexicales. Ces exemples démontrent que la créativité lexicale n'est pas l'apanage des experts ou des écrivains mais est au contraire une faculté humaine partagée par tous les locuteurs d'une langue. Judith Munat conclut que la créativité lexicale prend des formes variées, remplit des fonctions diverses et mérite d'occuper une place centrale dans la recherche en linguistique. television and morphological inventiveness » (p. 159-171), prend pour point de départ 
la distinction entre créativité et inventivité : la première, selon lui, découle de l'application de règles productives, tandis que la seconde est l'appropriation individuelle de la créativité et conduit à un résultat innovant et apprécié des autres locuteurs. C'est à la lumière de cette distinction qu'il se propose d'étudier deux phénomènes qu'il retient en raison de l'enthousiasme qu'ils ont suscité auprès de très jeunes téléspectateurs de programmes pour enfants : la formation «ninky nonk », dont il traite brièvement et qui met en œuvre une réduplication avec changement vocalique, puis ce qu'il nomme la "mucker upper formation ", qu'il analyse en détail et qui consiste en la double suffixation par -er d'un verbe à particule pour former un nom d'agent. Jim Walker cherche d'abord à déterminer la productivité de ce phénomène : une recherche sur Google et GoogleBooks lui permet de conclure qu'il ne s'agit pas d'une forme rare en voie d'extinction. Il s'aperçoit que les formes récurrentes, dans leur majorité, dérivent de verbes qui se composent de deux formes monosyllabiques et dont la particule adverbiale se termine par une occlusive (act out, add up, act up, ask out). Les données de l'Oxford English Dictionary corroborent ces résultats. Jim Walker remarque, en se fondant sur le corpus TIME (qui se constitue d'articles du magazine Time publiés depuis les années 1920) que c'est dans les années 1940 que ces formes sont les plus fréquentes. Leur emploi diminue par la suite jusqu'aux années 2000, où elles connaissent un regain marqué.

L'auteur en déduit que ces formes sont «créatives » et non "inventives » puisqu'elles correspondent à l'application d'une règle morphologique. Toutefois, les dérivés présentent une alternance entre syllabes fortes et syllabes faibles et se caractérisent par leur euphonie : à ce titre, on peut considérer les deux phénomènes étudiés par Jim Walker comme «inventifs" puisque les locuteurs font ceci d'innovant qu'ils ont recours au "quasi-morphème»-er comme outil d'euphonie. L'auteur en déduit que l'euphonie mériterait que la recherche en linguistique lui accorde une place plus importante.

Dans « Euphemisms for death: reinventing reality through words?» (p. 173-187), Denis Jamet s'intéresse aux euphémismes employés pour renvoyer à la mort dans la série Six Feet Under.

50 Il rappelle que la mort est un tabou depuis l'ère victorienne et que c'est l'une des raisons pour lesquelles il est difficile de la nommer directement: les euphémismes constituent une sorte de bouclier protecteur contre une réalité crainte et déplaisante. Ils correspondent donc à un «comportement verbal» (cf. E. Crespo Fernández [2005: 78]) et non à un simple choix lexical. L'hypothèse avancée par Denis Jamet est qu'ils permettent même de réinventer la réalité. Toutefois, plus les euphémismes deviennent conventionnels, moins ils sont efficaces comme protection contre la réalité menaçante qu'ils désignent. Ainsi, «untertaker», qui était initialement un euphémisme, a été remplacé par un autre euphémisme, « mortician », qui a à son tour été supplanté par « funeral director».

Denis Jamet s'aperçoit que dans Six Feet Under, les euphémismes reposent sur :

- des termes techniques, des emprunts (au français et au latin, surtout) et des mots savants : " expire ", « necrophilia ", « mortuary », « the deceased », etc., qui mettent à distance la réalité désignée ;

- des circonlocutions («memorial park» / «garden of remembrance» pour " cimetière ", « human remains ", « death-care facilities »), qui diluent l'effet menaçant de la mort ; 
- des acronymes («he OD’ed»), qui « découpent» le signifié pour le rendre moins choquant ;

- l'effacement ou l'omission (comme dans l'énoncé suivant, où «burial» est omis : « What did you have in mind for your son's... »);

- l'hyperonymie ou la métonymie: (dans l'énoncé "You met him at Dad's thing», « thing » se substitue à « death ceremony »);

- des métaphores, qui permettent de s'approprier l'inconnu qu'est la mort en l'assimilant à des domaines connus qui ont souvent des connotations positives. Parmi ces métaphores, on peut citer LA MORT EST UN VOYAGE ( « Your daddy has gone away for a very long time ", «I'm getting him ready so his family can see him [...] and say goodbye to him »), LA MORT EST REPOS/SOMMEIL (« He looks like he is sleeping»), LA MORT EST LA FIN («It will not bring her back»), LA MORT EST UNE PERTE («I'm very sorry about your loss »).

Denis Jamet revient sur son hypothèse de départ, selon laquelle les termes et expressions que nous utilisons pour parler de la mort sont euphémistiques: les euphémismes employés sont en réalité la façon habituelle, normale, communément acceptée de parler de la mort et ils érigent un mur virtuel et fictionnel entre la réalité et la perception que nous en avons.

Dans « "Mad I am not"... or am I? Poe's voices of Madness » (p.189-200), Jocelyn Dupont s'intéresse à la façon dont Edgar A. Poe traite de la maladie mentale dans "Berenice " (1835), « The Tell-Tale Heart » (1843) et « The Black Cat » (1843). Il remarque que Poe ne connaissait pas véritablement la science psychiatrique de son époque et que son approche de la maladie mentale est avant tout poétique et littéraire.

Jocelyn Dupont note que bien que le narrateur de «Berenice» soit fou, ni l'adjectif « mad» ni le substantif «madness » ne figurent dans le texte. "The Black Cat », écrit huit ans après «Berenice ", témoigne de l'évolution de la façon dont Poe fait référence à la folie : le mot « mad » apparaît cette fois-ci dès les premières lignes :

For the most wild, yet most homely narrative which I am about to pen, I neither expect nor solicit belief. Mad indeed would I be to expect it [...]. Yet, mad am I not and very surely do I not dream. [E. A. Poe 1994 (1843) : 563]

L'inversion de "mad am I not " produit, selon Jocelyn Dupont, une sorte de double négation puisqu'elle annule le déni de folie.

"The Tell-Tale Heart » témoigne d'un changement de stratégie évident par rapport à «Berenice » et « The Black Cat », ce qui est perceptible dès l'incipit :

True! - nervous - very, very dreadfully nervous I had been and am; but why will you say that I am mad? The disease had sharpened my senses - not destroyed - not dulled them. [E.A. Poe 1994 (1843) : 313]

Comme dans "The Black Cat ", il est d'autant plus clair que le narrateur est fou qu'il ne cesse de le nier, mais les phrases sont ici très courtes et une énergie absente des deux autres contes se dégage de ces lignes. Le rythme des phrases semble calquer celui des battements du cœur du narrateur (cf. E. Phillips [1979:142]) : l'inventivité de Poe consiste ici à mimer dans la diégèse une entropie interne. Le narrateur s'adresse également fréquemment au lecteur pour le convaincre qu'il n'est pas fou, ce qui tend à prouver l'inverse: "If you still think me mad, you will think it no longer when I describe the wise precautions I took for the concealment of the body» [E. A. Poe 1994 (1843) : 316].

De plus, les répétitions abondent : 
I moved it slowly - very, very slowly [E. A. Poe 1994 (1843) : 313]

I resolved to open a little - very, very little crevice [E. A. Poe 1994 (1843) : 315]

Ces répétitions semblent figurer l'enfermement mental du protagoniste, pris au piège de sa propre folie, et la violence de sa voix intérieure. Le texte n'est plus une confession tourmentée, comme dans «Berenice ", voire dans « The Black Cat » : on assiste plutôt à une représentation linguistique brute et dynamique de la folie.

Jocelyn Dupont insiste en conclusion sur le contexte intellectuel de ces textes de Poe, puisque ses contes datent de la période où la psychiatrie émerge comme science. Poe, avant tout poète, aborde la folie dans une perspective intuitive et esthétique. Par ailleurs, il écrit durant la première moitié du XIXe siècle, avant l'invention de l'inconscient, c'est donc le conscient qui règne en maître dans ses écrits et non les profondeurs enfouies de la psyché.

Dans la conclusion (p. 201-207), Sandrine Sorlin trace les contours que pourrait prendre une linguistique inventive. Elle souligne que les contributions qui composent l'ouvrage montrent tout l'intérêt de ne pas séparer une linguistique "sérieuse " d'une linguistique populaire, littéraire ou poétique. En effet, plusieurs articles témoignent de ce que des pratiques langagières qui paraissent a priori marginales ou déviantes (en littérature, notamment) mettent en réalité à nu le fonctionnement de la langue. Une linguistique inventive aurait précisément pour objectif de faire émerger ce qui est resté dans l'ombre et d'ouvrir de nouvelles pistes d'exploration, elle doit donc inclure ce qui peut perturber le fonctionnement de la langue et reconnaître une contradiction inhérente au langage: celle d'être produite par des règles qui sont elles-mêmes évolutives.

Plusieurs contributions concernent une autre face de la linguistique inventive : l'étude même de la créativité et de l'inventivité : Denis Jamet, par exemple, montre à quel point l'invention est nécessaire pour « désodoriser » la langue et produire une réalité acceptable par les sujets humains ; Laure Gardelle étudie des tentatives de renouveler la langue et les obstacles sur lesquels elles achoppent; John E. Joseph souligne, quant à lui, que l'inventivité n'est pas l'apanage du locuteur, dans la mesure où locuteurs et auditeurs en font également preuve.

60 Enfin, Sandrine Sorlin plaide pour une linguistique interdisciplinaire, qui consisterait non à convoquer quelques disciplines autour d'un même thème mais à « créer un objet nouveau, qui n'appartienne à personne»[R. Barthes 1984:106]. Elle rappelle que l'ouvrage constitue justement une première étape vers la possible réorganisation d'une linguistique qui étudierait ce nouvel objet à inventer ou à réinventer.

61 Les objets étudiés dans Inventive Linguistics sont d'une grande diversité: de l'épistémologie, à la littérature, en passant par les langues imaginaires, et les corpus employés ou analysés par les auteurs sont également très variés (poèmes, contes, romans, œuvres musicales, séries, etc.). L'inventivité et l'invention y sont examinées sous toutes leurs coutures et à différentes échelles; leur nécessité (celui du renouvellement de la langue, effectif ou visé) est prise en considération, tout comme leurs limites. L'inventivité en linguistique et par la linguistique n'est pas non plus oubliée. Par ailleurs, les différents contributeurs font preuve d'inventivité, en s'intéressant à ce qui a été laissé à la marge et en ouvrant des pistes théoriques innovantes : en ce sens, l'ouvrage renouvelle la linguistique et est à la fois théorique et pratique, conforme en cela à la linguistique inventive que Sandrine Sorlin appelle de ses vœux. L'intérêt de cet ouvrage réside donc non seulement dans la richesse, la 
densité et l'originalité de chacune de ces contributions mais aussi dans le fait qu'il illustre de façon convaincante les pistes théoriques qu'il ouvre.

\section{BIBLIOGRAPHIE}

BARTHES Roland, 1984, Le Bruissement de la langue, Paris : Editions du Seuil.

BECKETT Samuel, 1961, Comment c'est, Paris : Minuit.

BUTLER Judith, 1997, Excitable Speech, London: Routledge.

CARRUTHERS Mary, 2000, The Craft of Thought: Meditation, Rhetoric, and the Making of Images, 400-1200, Cambridge: Cambridge University Press.

CH'IEN Evelyn, 2004, Weird English, Harvard: Harvard University Press.

Dickow Alexander, 2008, Caramboles, Paris : Argol.

CRESPO FERNÁNDEZ Eliecer, 2005, "Euphemistic strategies in politeness and face concerns", in Pragmalingüistica 13, Universidad de Cádiz, 77-86.

Foucault Michel, 1994 (1969), « Qu'est-ce qu'un auteur ?», Dits et écrits (1994), Paris : Gallimard, 789-809.

HocKETT Charles, 1958, A Course in Modern Linguistics, New York: Macmillan.

JACKSON Laura (Riding) \& JACKSON Schuyler B., 1997, Rational Meaning: A New Foundation for the Definition of Words, and Supplementary Essays, Charlottesville: University Pres of Virginia.

LECERCLE Jean-Jacques, 2004, Une philosophie marxiste du langage, Paris : PUF.

MuNAT Judith (Ed.), 2007, Lexical Creativity, Texts and Contexts, Amsterdam: John Benjamins.

PHILlIPS Elizabeth, 1979, Edgar Allan Poe: An American Imagination, Port Washington: Kennikat Press.

POE Edgar Allan, 1994 (1843), Tales of Mystery and Imagination, Clarke Graham (Ed.), London: Everyman.

PONGE Francis, 2002 (1971), La Fabrique du Pré. Euvres complètes II, Paris : Gallimard, 423-517.

PYNCHON Thomas, 1997, Mason \& Dixon, New York: Henry Holt.

VAugElas Claude Favre de, 1934 (1647), Remarques sur la langue françoise, Streicher J. (Ed.), Paris :

Droz.

WENDT Albert, 2003, The Mango's Kiss, New Zealand: Vintage. 


\section{AUTEURS}

\section{STÉPHANIE BÉLIGON}

Stéphanie Béligon, Sorbonne Université, Centre de Linguistique en Sorbonne (CeLiSo - UR 7332), France.

Stéphanie Béligon est Maître de conférences en linguistique anglaise à Sorbonne Université. Ses recherches portent sur la sémantique lexicale et sur l'expression de la perception et des émotions. 